\title{
Direct Formation of Catalyst-Free Zno Nanobridge Devices on an Etched Si Substrate Using a Thermal Evaporation Method
}

\author{
Jong Soo Lee, ${ }^{\dagger}$ M. Saif Islam, ${ }^{\ddagger}$ and Sangtae $\mathrm{Kim}^{\star}, \dagger$ \\ Department of Chemical Engineering and Materials Science, University of California, \\ Davis, California 95616, and Department of Electrical and Computer Engineering, \\ University of California, Davis, California 95616
}

Received April 19, 2006

\begin{abstract}
Well-aligned single crystalline $\mathrm{ZnO}$ nanobridges have been synthesized selectively across the prefabricated electrodes on silicon substrates by a single-step thermal evaporation method without using any metal catalysts or a predeposited ZnO seed layer that was a prerequisite for such synthesis. The growth region was self-defined by the anisotropic surface of the silicon substrate as initially postulated. Careful control of the reaction time and the substrate temperature allows the nanobridges to form almost exclusively across the electrodes. The photoresponses as well as the current-voltage characteristics of the device confirm that this single-step process indeed offers a simple and a cost-effective way to integrate self-assembled nanodevices based on individual and/or a large number of ZnO nanowires with conventional circuits without using e-beam lithography techniques and/or additional costly deposition processes.
\end{abstract}

In recent years, a large number of one-dimensional nanostructures have been synthesized with precisely controlled chemical compositions, morphologies, and sizes using various synthesis methods for potential applications in a variety of nanoscale devices. These include transistors, memory devices, logics, chemical/biosensors, and lasers. ${ }^{1-4}$ In view of various distinctive properties of $\mathrm{ZnO}$ (a direct band gap of $3.37 \mathrm{eV}$ at room temperature), nanodevices based on this material hold a lot of promises for, in particular, gas-sensing, electronic, optoelectronic applications. ${ }^{5-8} \mathrm{ZnO}$-based nanodevices are also promising candidates for biological applications due to easy fabrication and friendly nature to living organisms. ${ }^{9}$

Electrical and optical properties of nanowires are typically measured by making metallic (ohmic) contacts to the nanowires placed on an insulating substrate. To fabricate such devices, nanowires are usually separated from a substrate on which the nanowires were initially grown and then are randomly dispersed onto a desired insulating substrate after being diluted in a solution. In most cases, an electron beam lithography technique is applied to interface the nanowires with other elements of a circuit or a system. ${ }^{5,6,10,11}$ Although this research-based approach is suitable for a single or few

* Corresponding author. E-mail: chmkim@ucdavis.edu. Phone: (530) 754-2254. Fax: (530) 752-9554.

Department of Chemical Engineering and Materials Science.

$\doteqdot$ Department of Electrical and Computer Engineering. device demonstrations, it is rather complicated and timeconsuming such that it may not be an economically attractive integration process for conventional complementary metal oxide semiconductor compatibility.

On the other hand, for accurate measurements of the intrinsic properties, the nanowires need to be free from a contact with any object and to be surrounded by nonconductive material to eliminate possible artifacts attributed to current paths other than those through the nanowires themselves. ${ }^{6}$ Placing nanowires on an insulating surface, however, cannot completely eliminate the unwanted current paths. An ideal solution to this problem is direct lateral growth of suspended nanowires between two prefabricated electrodes. Recently Islam et al. introduced a bridging technique that connects a large number of highly oriented, suspended metal-catalyzed silicon nanowires between two electrodes to design nanodevices that could be easily integrated with modern electronic fabrication technologies. ${ }^{12}$

Although catalysts help in the synthesis of various nanowires, they may also add impediment by becoming an impurity introduced into the nanowire structure. Often these types of impurities can hinder the performance of a device made with the nanowires. For example, one of the known issues a gold $(\mathrm{Au})$ nanoparticle used for growing semiconductor nanowires is the near-center band gap ionization energy level when $\mathrm{Au}$ is present in silicon. ${ }^{13}$ Some metals 
such as $\mathrm{Au}$ and $\mathrm{Cu}$ contribute to a high level of generationrecombination currents if the metals are present in the semiconductor, even if the level of the metal is almost undetectable. Thus it is desirable to develop techniques for synthesizing nanowires without any metal catalysts.

Selective growth of $\mathrm{ZnO}$ nanorods on various substrates without using metal catalysts has been reported. ${ }^{14-19}$ Very recently Conley et al. used a predeposited thin film $\mathrm{ZnO}$ layer alternative to metal catalysts to define the regions of nanowire growth. ${ }^{18}$ Deposition of the seed layer on a Si substrate using an atomic layer deposition technique was thus a prerequisite to grow suspended $\mathrm{ZnO}$ nanowires between two electrodes in their work. This rather costly process, however, may not be necessary to selectively form $\mathrm{ZnO}$ nanobridges across the electrodes on Si substrates.

Surface morphology of an anisotropic crystalline substrate plays a major role in controlling the growth kinetics of lowdimensional structures. ${ }^{20}$ The anisotropy of the surface often influences the growth of nanowires since it can induce an anisotropic strain and, thus, can confine the adatom diffusion to one dimension. ${ }^{21}$ Nucleation usually occurs in sequence at edges, at steps, and on planar surfaces in order to reduce the surface energy. ${ }^{22,23}$ The higher the density of broken bonds in the region, the higher the surface energy. Therefore it is expected that the edges of the prefabricated Si electrodes on a Si substrate may provide the most favorable site for a vapor to bond in a vapor-solid process since the edges of the electrodes serve as higher-energy binding sites. Consequently, the nature of structures of the electrodes on $\mathrm{Si}$ substrate alone may prevent random growth of $\mathrm{ZnO}$ on a $\mathrm{Si}$ substrate and define the favorable growth regions such that suspended $\mathrm{ZnO}$ nanobridges are expected to form preferentially across the electrodes even without a seed layer.

In this Letter we report a direct single-step method that $\mathrm{ZnO}$ nanowires selectively bridge prefabricated $\mathrm{Si}$ electrodes on a Si substrate without using any metal catalysts or a seed layer to avoid interaction of the surface of the nanowires with the surroundings. We also demonstrate that the device fabricated by using this method exhibits high photosensitivity required for optoelectronic devices.

$\mathrm{ZnO}$ nanowires were grown on two different types of substrates: on-axis $\mathrm{Si}(100)$ wafers ( $\mathrm{Si}-1 \mathrm{~s})$ and etched $\mathrm{Si}(110)$ wafers with exposed $\mathrm{Si}(111)$ vertical planes bounding trenches $(\mathrm{Si}-2 \mathrm{~s}){ }^{12}$ The nanowires grown on $\mathrm{Si}-1 \mathrm{~s}$ were to be used for the chemical and the structural chracterizations (see below) while $\mathrm{Si}-2 \mathrm{~s}$ were to form $\mathrm{ZnO}$ nanobridges across the trenches. The trenches are approximately $10-13 \mu \mathrm{m}$ deep and $2-6 \mu \mathrm{m}$ wide.

The $\mathrm{ZnO}$ nanowires were grown in an alumina tube horizontally placed in a conventional tube furnace. An alumina boat containing pure $\mathrm{ZnO}$ powders (Aldrich, 99.99\%) was loaded into the center of the alumina tube. Si-1s and $\mathrm{Si}-2 \mathrm{~s}$ were placed in pairs at the several different temperature zones nearer to the downstream end of the tube to ensure that the $\mathrm{ZnO}$ nanowires and the nanobridges grown on $\mathrm{Si}$ $1 \mathrm{~s}$ and $\mathrm{Si}-2 \mathrm{~s}$, respectively, are structurally as well as chemically identical. The $\mathrm{ZnO}$ powders were thermally evaporated at $1450{ }^{\circ} \mathrm{C}$ with an argon flow under a constant
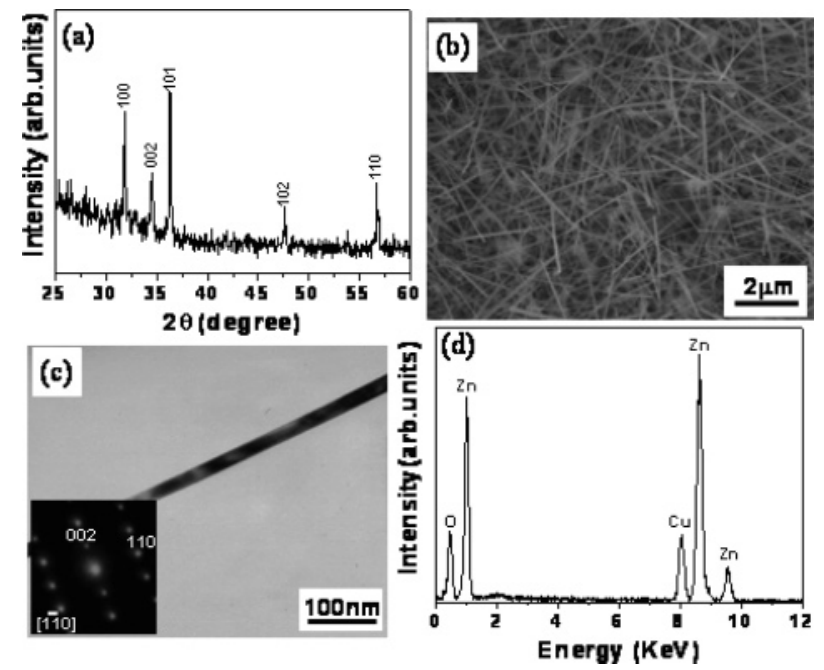

Figure 1. An XRD pattern (a), a FE-SEM image (b), a bright field TEM image (c), and an EDX spectrum (d) of $\mathrm{ZnO}$ nanowires synthesized on the on-axis $\operatorname{Si}(100)$ substrate at $850{ }^{\circ} \mathrm{C}$ for $120 \mathrm{~min}$.

furnace chamber pressure of $0.7 \mathrm{~atm}$. The evaporation time and the flow rate were precisely controlled. The temperature of the substrates varied from 600 to $850^{\circ} \mathrm{C}$ depending upon their locations in the alumina tube.

The chemical and the structural analyses on as-synthesized $\mathrm{ZnO}$ nanowires on $\mathrm{Si}-1 \mathrm{~s}$ were carried out using a X-ray diffractometer (XRD, Scintag XDS-2000) with $\mathrm{Cu} \mathrm{K \alpha}$ radiation, a field emission scanning electron microscope (FESEM, FEI XL-30 SFEG), and a transmission electron microscope (TEM, Philips CM-12).

Figure 1 summarizes the results for the $\mathrm{ZnO}$ nanowires grown on a Si-1 at $850{ }^{\circ} \mathrm{C}$ for 120 min with the argon flow rate of 200 standard cubic centimeters per minute $(\mathrm{sccm})$. The measured XRD peaks are indexed to confirm the wurtzite hexagonal structure (Figure 1a). The diameters of the nanowires range from 20 to $150 \mathrm{~nm}$, and their lengths are found to be several micrometers as shown in a FE-SEM image (Figure 1b). Parts $\mathrm{c}$ and $\mathrm{d}$ of Figure 1 reveal a representative TEM image (Figure 1c) and an energydispersive X-ray (EDX) spectrum (Figure 1d), respectively, of one of the nanowires shown in Figure $1 b$ with a diameter of about $30 \mathrm{~nm}$. The electron diffraction pattern of the nanowire (see the inset of Figure 1c) clearly demonstrates that the synthesized nanowires are in a single crystalline phase. The chemical composition of the $\mathrm{ZnO}$ nanowire was confirmed by the peaks associated with $\mathrm{Zn}$ and $\mathrm{O}$ atoms shown in Figure 1d. (The Cu-related peak in the spectrum comes from the $\mathrm{Cu}$ grids.)

Figure 2 shows the sequential FE-SEM images of the formation of nanobridges across the electrodes on $\mathrm{Si}-2 \mathrm{~s}$ in $600{ }^{\circ} \mathrm{C}$ (Figure $2 \mathrm{a}-\mathrm{c}$ ) and $850{ }^{\circ} \mathrm{C}$ (Figure $2 \mathrm{~d}, \mathrm{e}$ ) zones. In view of Figure 1 together with the fact that these nanobridges are formed under the identical conditions under which the nanowires shown in Figure 1 were grown on $\mathrm{Si}-1 \mathrm{~s}$ as described above, it is reasonable to assume that these nanobridges are chemically as well as structurally identical to the nanowires shown in Figure 1. Note that neither metal catalysts nor a predeposited $\mathrm{ZnO}$ seed layer was used to form these nanobridges across the electrodes on a Si substrate. 


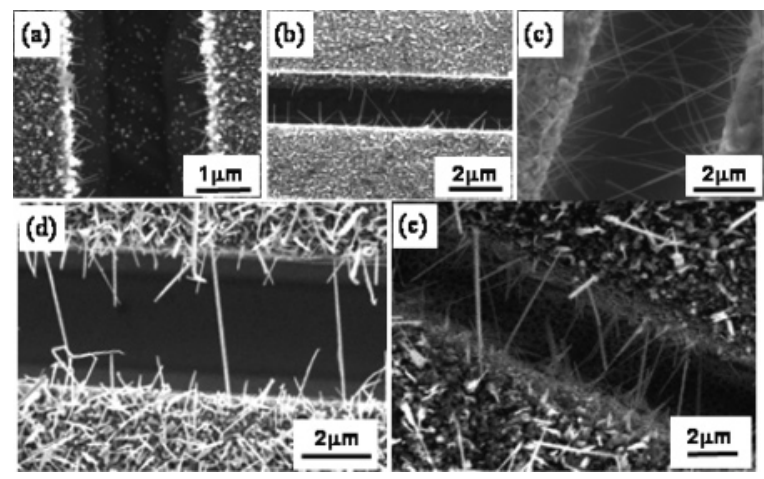

Figure 2. FE-SEM images of $\mathrm{ZnO}$ nanobridges formed across two electrodes at $600{ }^{\circ} \mathrm{C}$ for $20 \mathrm{~min}$ (a), $60 \mathrm{~min}$ (b), and $120 \mathrm{~min}$ (c) and at $850{ }^{\circ} \mathrm{C}$ for $60 \mathrm{~min}$ (d) and $120 \mathrm{~min}$ (e), respectively.

Figure 2a shows a representative image of a sample that was collected in the $600^{\circ} \mathrm{C}$ zone at the reaction time of 20 min. $\mathrm{ZnO}$ nanowires start to laterally grow from the vertical walls ( $\mathrm{Si}(111)$ plane) of a trench etched on a $\mathrm{Si}(110)$ substrate toward the opposing vertical surfaces. Nearly no sign of nanowire growth was observed before 20 min of the reaction time while nanopowders were formed on the surface of the electrodes in this temperature zone. The density of the powder was found to be distinctively higher near the edges of the electrodes as clearly seen in Figure 2a. EDX analysis confirmed that the chemical composition of these powders is $\mathrm{ZnO}$. Figure 2a also demonstrates that $\mathrm{ZnO}$ nanowires start to preferentially grow from the edge areas. As reaction time reaches $60 \mathrm{~min}$ (Figure 2b), the nanowires shown in Figure 2a further grow and impinge on the opposing vertical surface to form nanobridges. A majority of nanowires are found to be still almost exclusively formed across the two electrodes. The density of the nanobridges becomes significantly higher when the reaction time was increased to 120 min (Figure 2c). At this reaction time, however, the regional selectivity of the formation of $\mathrm{ZnO}$ nanowires on the $\mathrm{Si}-2 \mathrm{~s}$ starts to become lower.

On the other hand, in the $850{ }^{\circ} \mathrm{C}$ zone (parts $\mathrm{d}$ and e of Figure 2), within $60 \mathrm{~min}$ (Figure 2d), much denser and thicker $\mathrm{ZnO}$ nanobridges compared to those shown in Figure $2 \mathrm{~b}$ are formed and a significant number of the nanowires have already started to grow on the top surface of the electrodes. Figure $2 \mathrm{e}$ shows an image obtained from a sample that was in this temperature zone for $120 \mathrm{~min}$.

We have initially postulated that the evaporated $\mathrm{ZnO}$ preferentially nucleates near the edge area of the prefabricated electrodes on a Si substrate to self-define the growth area as schematically sketched in Figure 3a,b. Such higher density of $\mathrm{ZnO}$ nanopowder deposited near the edges of the electrodes is expected to allow $\mathrm{ZnO}$ nanowires to first grow from those areas to bridge the two electrodes (Figure 3c). Consequently, as the growth continues, the density of $\mathrm{ZnO}$ nanowires grown across the two electrodes becomes distinctively higher than that of $\mathrm{ZnO}$ nanowires grown at the plane surface (Figure 3c). Figure 2 indeed strongly supports this postulate.

Figure 4a shows $\mathrm{ZnO}$ nanobridges synthesized under the optimized reaction conditions $\left(850^{\circ} \mathrm{C}\right.$ for $40 \mathrm{~min}$ ). $\mathrm{ZnO}$

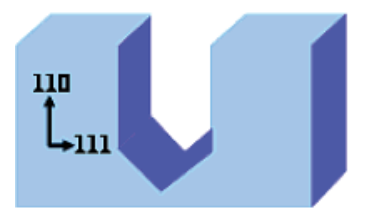

(a)

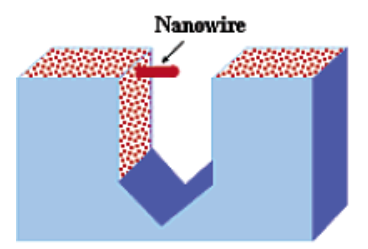

(c)

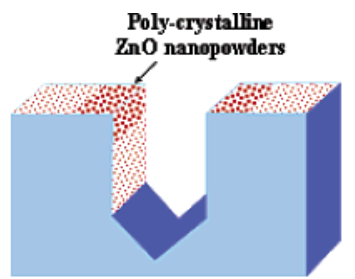

(b)

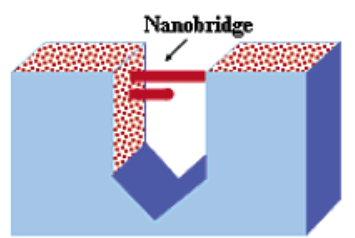

(d)
Figure 3. Schematic sketches of (a) prefabricated two electrodes on a $\mathrm{Si}$ substrate, (b) nucleation of $\mathrm{ZnO}$ nanopowders on the substrate, (c) $\mathrm{ZnO}$ growing across the two electrodes, and (d) a formation of $\mathrm{ZnO}$ nanobridges.

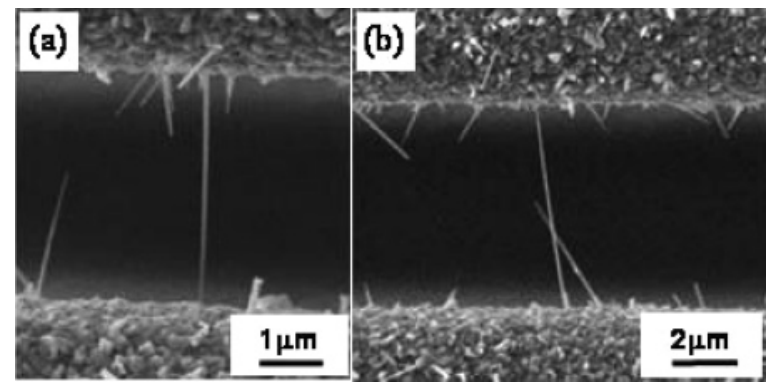

Figure 4. $\mathrm{ZnO}$ nanobridges formed across two electrodes with a separation of $4 \mu \mathrm{m}$ (a) and $6 \mu \mathrm{m}$ (b) at $850{ }^{\circ} \mathrm{C}$ for $40 \mathrm{~min}$, respectively.

nanobridges were formed almost exclusively across the two electrodes. The distances between the two electrodes are 4 $\mu \mathrm{m}$ (Figure 4a) and $6 \mu \mathrm{m}$ (Figure $4 \mathrm{~b}$ ). It is thus confirmed that $\mathrm{ZnO}$ nanobridges can be directly as well as selectively formed across the prefabricated electrodes on Si substrate by a single-step thermal evaporation method without using metal catalysts or a predeposited seed layer.

Figure 5 shows the current - voltage $(I-V)$ characteristics of the synthesized $\mathrm{ZnO}$ nanobridges on a silicon-on-insulator (SOI) substrate in the dark and under illumination of UV light $(\lambda=365 \mathrm{~nm})$. The measurements were performed on the device in air by probing directly on the Si electrodes without depositing metal for better contacts. The distance between electrodes was $4 \mu \mathrm{m}$. Figure 5 clearly demonstrates typical nonlinear and asymmetric $I-V$ characteristics of both the dark current and the photocurrent which can be attributed to the contact barriers existing between $\mathrm{Si}$ and $\mathrm{ZnO}$ nanobridges. As seen in Figure 5, under UV irradiation, the measured current was increased from 0.014 to $2.21 \mu \mathrm{A}$ at the applied voltage of $5.0 \mathrm{~V}$. Such a high increase in the current under the UV irradiation indicates the higher sensitivity of this device to UV light compared with other $\mathrm{ZnO}$ nanowire devices previously reported. ${ }^{18,24-26}$

Finally, the time-dependent photoresponses of the $\mathrm{ZnO}$ nanobridges in air at a bias voltage of $2.0 \mathrm{~V}$ are shown in 


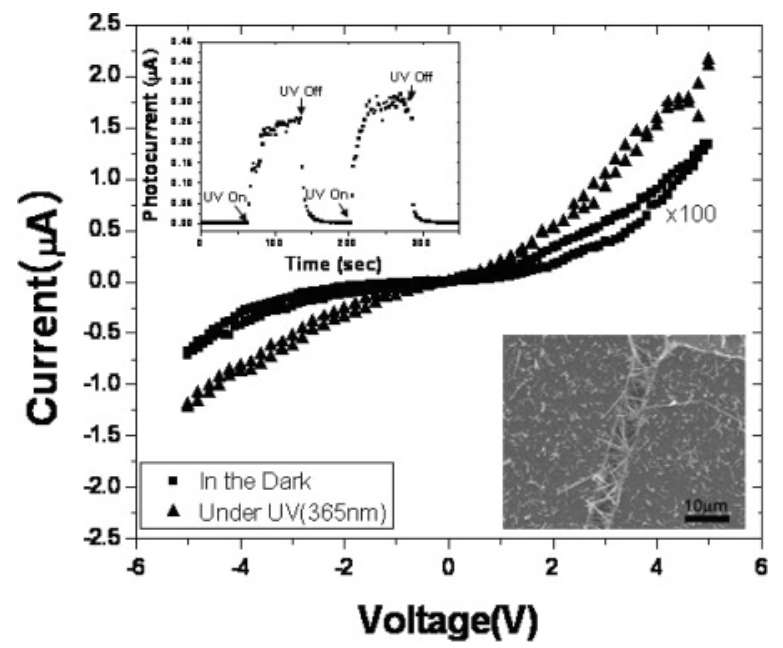

Figure 5. $I-V$ characteristics of the dark current and the photocurrent (under $365 \mathrm{~nm}$ ) of $\mathrm{ZnO}$ nanobridges on a silicon-oninsulator (SOI). (The inset shows the time-dependent photoresponses of the device in air at a bias voltage of $2.0 \mathrm{~V}$.)

the inset of Figure 5. When the UV lamp was switched on, the current sharply increased to $0.20 \mu \mathrm{A}$ at about $2.7 \mathrm{~s}$ and then reached the saturation value of $0.27 \mu \mathrm{A}$ within $18 \mathrm{~s}$. As soon as the UV light was turned off, the measured current drops down to $0.08 \mu \mathrm{A}$ at about $3.0 \mathrm{~s}$ and then further decreased to the initial value of $0.004 \mu \mathrm{A}$ within $50 \mathrm{~s}$. The observed photoresponses of the $\mathrm{ZnO}$ nanobridges can be attributed to adsorption and photodesorption of oxygen on the surface of the nanobridges in addition to rapid changes in conductivity by photogenerated electron-hole pairs. ${ }^{25-27}$

In summary, chemically pure single crystalline $\mathrm{ZnO}$ nanowires have selectively bridged across the prefabricated electrodes on silicon substrates by a single-step thermal evaporation method without using any metal catalysts or a predeposited $\mathrm{ZnO}$ seed layer. The results confirm the postulate that the growth region is self-defined by the anisotropic surface of the silicon substrate used. The $\mathrm{ZnO}$ nanowires almost exclusively grow near the edges of the electrodes to form the nanobridges across the electrodes under precisely controlled reaction conditions. The photoresponses of a $\mathrm{ZnO}$ nanobridge device fabricated in this study demonstrate that this single-step method is indeed proved to be a simple and cost-effective way that one can integrate self-assembled nanodevices based on individual and/or a large number of $\mathrm{ZnO}$ nanowires with conventional circuits without using e-beam lithography techniques and/or additional costly deposition processes.

Acknowledgment. J.S.L. acknowledges partial support from the Korea Research Foundation Grant funded by Korea
Government for this work (MOEHRD, Basic Research Promotion Fund, KRF-2005-214-D00305). Authors appreciate the help of Chad Johns for obtaining SEM images at the earlier stage of this work and of Ibrahim Kimukin for Si substrate preparation.

Note Added after ASAP Publication. This article was published ASAP on June 8, 2006. A name in the Acknowledgment was changed. The revised article was reposted on June 23, 2006.

\section{References}

(1) Fan, Z.; Lu, J. G. Appl. Phys. Lett. 2005, 86, 123510.

(2) Li, Q. H.; Liang, Y. X.; Wan, Q.; Wang, T. H. Appl. Phys. Lett. 2004, 85, 6389 .

(3) Yatsui, T.; Lim, J.; Ohtsu, M.; An, S. J.; Yi, G. C. Appl. Phys. Lett. 2004, 85, 727.

(4) Li, Q. H.; Wan, Q.; Liang, Y. X.; Wang, T. H. Appl. Phys. Lett. 2004, 84, 4556.

(5) Wang, Z. L. Annu. Rev. Phys. Chem. 2004, 55, 159.

(6) Johnson, J. C.; Knutsen, K. P.; Yan, H.; Law, M.; Zhang, Y.; Yang, P.; Saykally, J. Nano Lett. 2004, 4 (2), 197.

(7) Maiti, A.; Rodriguez, J. A.; Law, M.; Kung, P.; Mckinney, J. R.; Yang, P. Nano Lett. 2003, 3 (8), 1025.

(8) Fan, Z.; Lu, J. G. J. Nanosci. Nanotechnol. 2005, 5, 1561

(9) Yi, G. C.; Wang, C.; Park, W. I. Semicond. Sci. Technol. 2005, 20 (4), S22.

(10) Fan, Z.; Wang, D.; Chang, P. C.; Tseng, W. Y.; Lu, J. G. Appl. Phys. Lett. 2004, 85, 5923.

(11) Park, W. I.; Kim, J. S.; Yi, G. C.; Bae, M. H.; Lee, H. J. Appl. Phys. Lett. 2004, 85, 5052.

(12) Islam, M. S.; Sharma, S.; Kamins, T. I.; Stanley Williams, R. Nanotechnology 2004, 15, L5.

(13) Sze, S. M. Physics of semiconductor device; Wiley: New York, 1981.

(14) Chik, H.; Liang, J.; Cloutier, S. G.; Kouklin, N.; Xu, J. M. Appl. Phys. Lett. 2004, 84, 3376.

(15) Jie, J.; Wang, G.; Chen, Y.; Han, X.; Wang, Q.; Xu, B.; Hou, J. G. Appl. Phys. Lett. 2005, 86, 031909.

(16) Wang, L.; Zhang, X.; Zhao, S.; Zhou, G.; Zhou, Y.; Qi, J. Appl. Phys. Lett. 2004, 86, 024108.

(17) Conley, J. F., Jr.; Stecker, L.; One, Y. Nanotechnology 2005, 16, 292.

(18) Conley, J. F., Jr.; Stecker, L.; One, Y. Appl. Phys. Lett. 2005, 87, 223114.

(19) How Ng, T.; Han, J.; Yamada, T.; Nguyen, P.; Yi Chen, P.; Meyyappan, M. Nano Lett. 2004, 4(7), 1247.

(20) Himpsel, F. J.; Kirakosian, A.; Crain, J. N.; Lin, J. L.; Petrovykh, D. Y. Solid State Commun. 2001, 117, 149.

(21) Tersoff, J.; Tromp, R. M. Phys. Rev. Lett. 1993, 70, 2782

(22) Lee, Y. K.; Lee, M. S.; Lee, J. S. J. Cryst. Growth 2002, 244, 305.

(23) Walter, E. C.; Murray, B. J.; Favier, F.; Kaltenpoth, G.; Grunze, M.; Penner, R. M. J. Phys. Chem. B 2002, 106, 11407.

(24) Fan, Z.; Chang, P. C.; Lu, J. G.; Walter, E. C.; Penner, R. M.; Lin, C. H.; Lee, H. P. Appl. Phys. Lett. 2004, 85, 6128

(25) Ahn, S. E.; Lee, J. S.; Kim, H.; Kim, S.; Kang, B. H.; Kim, K. H.; Kim, G. T. Appl. Phys. Lett. 2004, 84, 5022.

(26) Kind, H.; Yan, H.; Messer, B.; Law, M.; Yang, P. Adv. Mater. 2002, 14,158

(27) Keezer, R. J. Appl. Phys. 1961, 35, 1866

NL060883D 